\title{
Applications cliniques des facteurs de croissance hématopoïétiques en onco-hématologie
}

Le génie génétique a permis le clonage des ADNc de nombreux interleukines et facteurs de croissance hématopoiétiques ainsi que la production de ces substances, permettant de tester leur utilité dans différentes conditions pathologiques. L'érythropoiétine est utile pour prévenir ou corriger l'anémie associée aux traitements des cancers. Le GM-CSF, l'interleukine 3 et le G-CSF sont l'objet d'essais thérapeutiques dans plusieurs situations : le traitement des syndromes myélodysplasiques, la potentialisation des traitements antileucémiques, la récupération des aplasies suivant les chimiothérapies et les greffes de moelle. Plusieurs résultats encourageants ont été obtenus. Les indications futures des facteurs de croissance hématopoiétiques devront néanmoins prendre en compte les possibles effets activateurs de ces substances sur la croissance des cellules malignes.

Jean-Dominique

Tigaud

Yves Bastion Bertrand Coiffier

\section{ADRESSES}

J.-D. Tigaud : chef de clinique des universités, assistant des hôpitaux. Laboratoire de cytologie analytique, universitć Claude-Bernard Lyon I, Domaine Rockef eller, 8 avenue Rockefeller, 69373 Lyon Cedex 08 et Service d'hématologie, centre hospitalier LyonSud, 69310 Pierre-Bénite, France.

Y. Bastion: chef de clinique des universités, assistant des hôpitaux. B. Coiffier : professeur des universités, praticien hospitalier. Service d'hématologie, centre hospitalier Lyon-Sud,

es facteurs de croissance hématopoïétiques (CSF pour colony-stimulating factors) sont des hormones glycoprotéiques qui contrôlent le fonctionnement de l'hématopoï̀se en agissant sur le couple proliférationdifférenciation des cellules souches médullaires jusqu'aux stades les plus matures des cellules sanguines. Ces cellules dérivent d'un stock de $10^{6}$ à $10^{7}$ cellules souches pluripotentes médullaires dont l'auto-renouvellement, la prolifération et la différenciation vers une lignée cellulaire sont contrôlés par ces CSF [1]. Les CSF stricto sensu sont des cytokines moléculairement identifiées ou en cours d'individualisation. En fait, la plu- part de ces cytokines participent au contrôle de l'hématopoïèse - soit directement, soit indirectement - en faisant intervenir des mécanismes à la fois cellulaires et humoraux, intriqués les uns avec les autres, aboutissant à une cascade d'effets biologiques (figure 1). C'est pourquoi la caractérisation du mécanisme précis du contrôle de l'hématopoïèse in vivo comme in vitro est très complexe, car il est le plus souvent difficile de déterminer avec certitude les effets propres de chaque molécule et de chaque type cellulaire impliqués [2, 3].

Schématiquement, on peut distinguer trois catégories de CSF hématopoïétiques en fonction de leurs principa- 


\section{* GLOSSAIRE *}

CSF : colony-stimulating factors, facteurs de croissance hématopoïétiques

EPO : érythropoiétine

G-CSF : granulocyte colony-stimulating factor

facteur stimulant la prolifération des granulocytes

M-CSF : macrophage colonystimulating factor

facteur stimulant la prolifération des macrophages

GM-CSF : granulocyte-macrophage colony-stimulating factor

facteur stimulant la prolifération des granulocytes et des macrophages

IL : interleukine

$\boldsymbol{I N F}$ : interféron

$\boldsymbol{L A} \boldsymbol{K}$ : lymphocytes activated killer lymphocytes tueurs activés

LAM : leucémies aiguës myéloïdes

LIF : leukemic inhibiting factor facteur inhibant les cellules leucémiques

SMD : syndromes myélodysplasiques

TNF : tumor necrosis factor

facteur de nécrose des tumeurs

CFU : colony forming units

Blast : cellules blastiques

$\boldsymbol{E}$ : cellules érythroïdes

GEMM : granulocytes, érythroblastes, macrophages, mégacaryocytes

Meg : mégacaryocytes

$M$ : macrophages

Eo : éosinophiles

Baso : basophiles

$G$ : granulocytes

GM : granulocytes et macrophages

$\boldsymbol{E}-\boldsymbol{B F U}$ : erythroid burst forming units précurseur des CFU-E

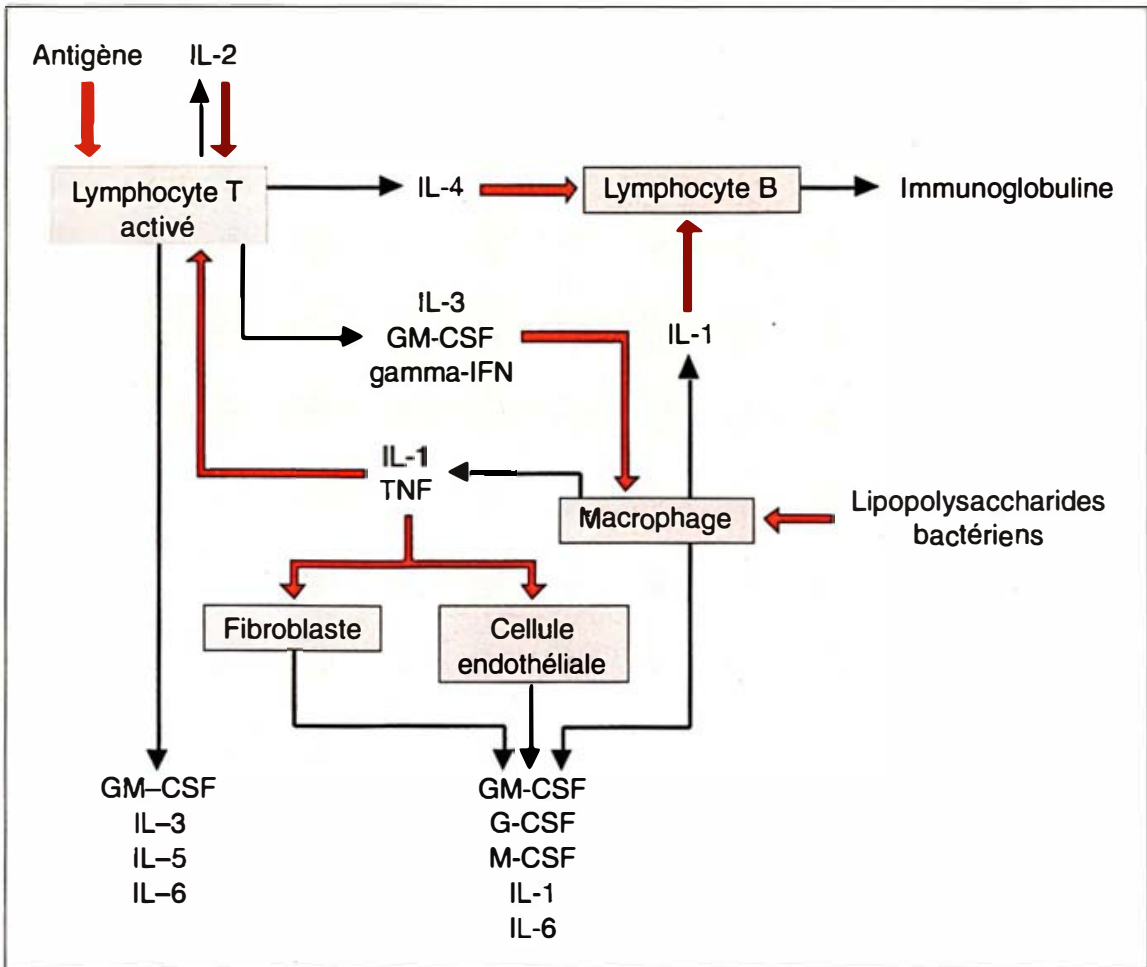

Figure 1. Représentation schématique de la cascade des effets biologiques en relation avec l'interaction des cytokines entre elles et avec leurs cellules cibles. (Pour la signification des abréviations, voir glossaire, ci-contre.)

les cellules cibles : le premier groupe comprend les cytokines agissant sur les stades les plus immatures des progéniteurs hématopoïétiques, pas tant en activant directement leur prolifération qu'en les sensibilisant aux autres CSF. Ainsi en est-il de l'interleukine 1 (IL-1), de l'interleukine 4 (IL-4), de l'interleukine 6 (IL-6) et de la human interleukin for $D A$ cells (HILDA) ou leukemic inhibiting factor (LIF). Le second groupe comprend des cytokines agissant sur des cellules moins immatures que les précédentes mais ayant gardé un potentiel de différenciation vers plusieurs lignées cellulaires différentes : l'interleukine 3 (IL-3) et le granulocytemacrophage colony-stimulating factor (GMCSF) stimulent des cellules pouvant se différencier en polynucléaires neutrophiles (PN), éosinophiles, ou basophiles, en monocytes, en mégacaryocytes ou en globules rouges. L'IL-3 est généralement plus active que le GM-CSF sur les précurseurs très indifférenciés, tandis que le GMCSF oriente principalement la différenciation cellulaire vers les lignées granuleuse et monocytaire. Le troisième groupe comprend les CSFs spécifiques d'une lignée de différenciation : le granulocyte colony-stimulating factor (G-CSF) et le macrophage colonystimulating factor (M-CSF) ont une action restreinte aux lignées respectivement granuleuse et monocytaire, l'interleukine 5 stimule l'éosinophilopoïèse et l'érythropoïétine (EPO) stimule la fabrication des globules rouges [4] (figure 2, p. 447). L'action de ces molécules passe par leur fixation à des récepteurs membranaires dont la caractérisation, actuellement en cours, montre l'hétérogénéité : non seulement il existe des récepteurs d'affinité différente pour un CSF donné sur une même population cellulaire [5], mais encore la densité de ces récepteurs sur la membrane cel- 


\section{RÉFÉRENCES}

1. Clark SC, Kamen $R$. The human hematopoietic colony-stimulating factors. Science 1987 ; 236 : 1229-37.

2. Sachs L. The control of growth and differentiation in normal and leukemic blood cells. Cancer $1990 ; 65$ : 2196-206.

3. Metcalf D. The colony-stimulating factors. Discovery, development, and clinical applications. Cancer $1990 ; 65$ : 2185-95.

4. Laver J, Moore MAS. Clinical use of recombinant human hematopoietic growth factors. J Natl Cancer Inst $1990 ; 81$ 1370-82.

5. Chiba S, Tojo A, Kitamura T, et al. Characterization and molecular features of the cell surface receptor for human granulocyte-macrophage colony-stimulating factor. Leukemia 1990 ; 4 : 29-36.

6. Piao YF, Okabe T. Receptor binding of human granulocyte colony-stimulating factor to the blast cells of myeloid leukemia. Cancer Res 1990 ; 50 : 1671-4.

7. Andreeff M, Welte K. Hematopoietic colony-stimulating factors. Semin Oncol $1989 ; 16: 211-29$

8. Nicola NA, Metcalf D, Matsumoto M, et al. Purification of a factor inducing differentiation in murine myelomonocytic leukemia cells : identification as granulocyte colony-stimulating factor. $J$ Biol Chem 1983 ; 258 : 9017-23.

9. Metcalf D. Regulator-induced suppression of myelomonocytic leukemic cells : clonal analysis of early cellular events. Int $J$ Cancer 1982 ; 30 : 203-10.

10. Begley CG, Metcalf D, Nicola NA. Purified colony-stimulating factors (G-CSF and GM-CSF) induce differentiation in human HL60 leukemic cells with suppression of clonogenicity. Int J Cancer 1987 ; 39 : 99-105

11. Metcalf D, Hilton DJ, Nicola NA. Clonal analysis of the actions of the murine leukemia inhibitory factor on leukemic and normal murine hemopoietic cells. Leukemia 1988 ; 2 : 216-21.

12. Shabo Y, Lotem J, Rubinstein M, et al. The myeloid blood cell differentiationinducing protein MGI-2A is interleukin 6 . Blood 1988 ; 72 : 2070-3.

13. Maekawa T, Metcalf D, Gearing DP Enhanced suppression of human myeloid leukemic cell lines by combinations of IL-6, LIF, GM-CSF and C-CSF. Int $J$ Cancer $1990 ; 45: 353-8$.

14. Souza LM, Boone TC, Gabrilove J, et al. Recombinant human granulocyte colonystimulating factor : effects on normal and leukemic myeloid cells. Science $1986 ; 232$ : 61-5.

15. Lista P, Brizzi MF, Avanzi G, et al Induction of proliferation of acute myeloblastic leukemia (AML) cells with hemopoietic growth factors. Leuk Res 1988 ; 12 lulaire dépend des cellules étudiées [6].

De fait, les CSF ont en général été introduits récemment dans l'arsenal thérapeutique onco-hématologique et leur diversité explique leurs très nombreuses indications (reconnues ou en cours d'investigation). Ainsi l'interleukine 2, seule ou en association avec les cellules lymphokine activated killer (LAK) est actuellement très largement prescrite pour son effet antitumoral - reconnu dans certaines indications, en cours d'expertise dans d'autres. D'autres cytokines, tels les interférons ou le tumor necrosis factor (TNF), sont en cours d'expertise. Les mécanismes d'action, les cellules effectrices, les effets biologiques élémentaires et leur action globale diffèrent significativement de ceux des CSF hématopoïétiques que nous étudierons donc en détail.

Principalement, cinq CSF hématopoiétiques, agissant très électivement et directement sur l'hématopoïèse, ont été largement étudiés chez l'homme. Les gènes et les $\mathrm{ADNc}$ de ces CSF ont été clonés et exprimés dans des champignons, des bactéries et des cellules eucaryotes. Les protéines recombinantes ainsi synthétisées ont été étudiées in vitro et in vivo, tant chez l'animal de laboratoire que chez l'homme. Il s'agit de l'IL-3, du GMCSF, du G-CSF, du M-CSF et de l'EPO [7] (Tableau I, p. 449).

Les CSF hématopoïétiques peuvent avoir des indications très variées : l'EPO est utilisé chez l'insuffisant rénal anémique, pour faciliter les autotransf usions ou corriger l'anémie des prématurés. Le G-CSF et le GM-CSF ont déjà montré leur efficacité dans les neutropénies congénitales sévères et le G-CSF dans les neutropénies cycliques. Par ailleurs, des essais contrôlés sont en faveur d'un rôle favorable de l'EPO, du GM-CSF et du G-CSF dans les anémies et infections des patients atteints de syndromes immuno-déficitaires acquis, notamment après traitement par la zidovudine (AZT).

Toutefois, c'est en onco-hématologie que les CSF hématopoïétiques paraissent d'ores et déjà avoir le plus large potentiel d'utilisation, comme en témoigne le grand nombre d'études cliniques menées aux États-Unis, en Europe et au Japon dans ce domaine. Deux types d'indications sont actuellement en cours d'expertise : d'une part, les maladies myéloïdes primitives aux premiers rangs desquelles les leucémies aiguës myéloïdes (LAM) et les syndromes myélodysplasiques (SMD), et, d'autre part, les insuffisances médullaires secondaires à des chimiothérapies cytotoxiques ou à des infiltrations tumorales.

\section{Leucémies aiguës myéloïdes et syndromes myélodysplasiques}

Dans ces cas, les CSF peuvent être utilisés soit pour leur action de différenciation des cellules clonales, soit pour leur action de potentialisation des chimiothérapies dépendantes du cycle en favorisant l'entrée en cycle de prolifération des cellules malignes quiescentes, soit enfin pour réduire la durée de l'aplasie post-chimiothérapique. Leur utilisation doit toutefois être très prudente car, si leur action sur le couple prolifération-maturation des cellules myéloïdes normales aboutit à une hématopoïèse équilibrée, leur action sur les cellules clonales, blastiques dans les LAM ou plus ou moins différenciées dans les SMD, fait courir le risque d'une accélération de la prolifération tumorale induite par les CSF.

\section{Leucémies aiguës myéloïdes}

Les nombreuses études conduites in vitro ont montré que la réponse des blastes myéloïdes aux CSF n'est pas univoque, mais dépend du type de facteur de croissance, du type d'hémopathie, des agents chimiques éventuellement associés et de la phase du cycle cellulaire dans laquelle se trouvent les cellules blastiques quand elles sont soumises à l'action des CSF. De nombreuses études ont déjà été rapportées dans la littérature concernant les différents effets des CSF hématopoïétiques sur les cellules blastiques.

- Effet de différenciation. En induisant l'engagement vers une lignée de différenciation puis en activant cette différenciation cellulaire, les CSF peuvent conduire des cellules blastiques à un stade très mature de différen- 


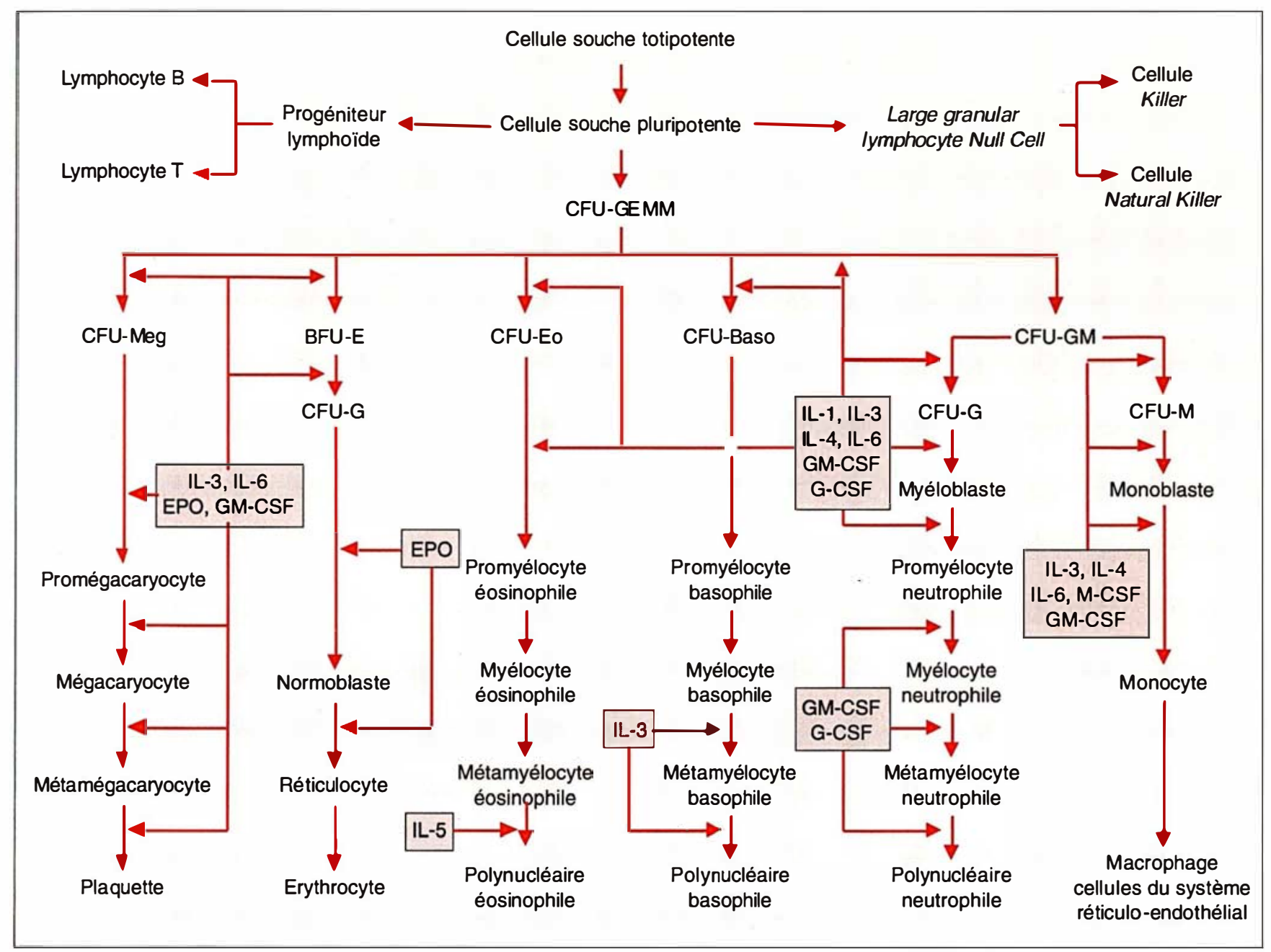

Figure 2. Principales cellules cibles des facteurs de croissance hématopoiétiques. (D'après [1.J) (Pour la signification des abréviations, voir glossaire, p. 445.)

ciation, leur enlevant leur caractère clonogène et les rendant incapables de proliférer. Le G-CSF s'est montré capable d'induire, après un effet initial prolifératif, la différenciation de la lignée leucémique WEHI-3B [8] jusqu'à un stade d'arrêt de la prolifération [9]. De même, le GCSF et le GM-CSF se sont montrés capables de supprimer la clonogénicité de la lignée leucémique myéloïde humaine HL60 par induction de différenciation [10]. Les CSF ne sont alors que des agents parmi d'autres susceptibles de bloquer des lignées cellulaires leucémiques : le LIF [11] ou l'interleukine 6 (IL-6) [12] seuls - ou en association avec les $m / s n^{\circ} 5$, vol. 7, mai 91
CSF, ont une telle action suppressive [13]. Les cellules leucémiques fraîches ne répondent que beaucoup plus rarement à cet effet de différenciation : ainsi, des concentrations élevées de G-CSF ajoutées au milieu de culture de cellules de plus de 50 leucémies aiguës fraîches n'entrâ̂nent une différenciation que dans $20 \%$ des cas, et encore, dans ces cas seulement $60 \%$ des cellules présentent des signes de différenciation [14].

- Effet de prolifération. C'est l'entrée en cycle de multiplication et la prolifération qui sont en fait le plus souvent observées ; l'index de marquage par la thymidine tritiée de cellules leucémiques myéloïdes humaines augmente dans la grande majorité des cas après exposition à l'IL-3, au GM-CSF et au G-CSF. De plus, la répétition des mesures a permis de mettre en évidence un recrutement progressif des cellules dans le cycle cellulaire [15]. Dans une autre étude, il est rapporté que, sur 35 cultures de cellules de LAM réalisées en méthylcellulose et en suspension, 34 prolifèrent en présence de G-CSF ou de GM-CSF. De façon remarquable, les concentrations en G-CSF ou GMCSF nécessaires pour avoir une réponse in vitro sont bien inférieures à celles que l'on doit atteindre pour faire répondre des cellules normales du même type [16]. 


\section{RÉFÉRENCES}

16. Murohashi I, Nagata K, Suzuki T, et al. Effects of recombinant G-CSF and GM$\mathrm{CSF}$ on the growth in methylcellulose and suspension of the blast cells in acute myeloblastic leukemia. Leuk Res 1988; 12 : 433-40.

17. Lista P, Porcu P, Avanzi GC, et al. Interleukin 3 enhances the cytotoxic activity of 1-B-D-arabinof uranosylcytosine (ara-C) on acute myeloblastic leukaemia (AML) cells. Br J Haematol $1988 ; 69$ : 121-3.

18. Büchner $T$, Hiddemann $W$, Koenigsmann $M$, et al. Recombinant human granulocyte-macrophage colony-stimulating factor after chemotherapy for acute leukemias at higher age or after relapse. In : Büchner, Schellong, Hiddemann, Ritter, eds. Haematology and Blood Transfusion, vol. 33, Acute Leukemias II. Berlin, Heidelberg : Springer-Verlag, 1990 : 724-31.

19. Estey EH, Dixon D, Kantarjian HM, et al. Treatment of poor-prognosis, newly diagnosed acute myeloid leukemia with Ara$\mathrm{C}$ and recombinant human granulocytemacrophage colony-stimulating factor. Blood $1990 ; 75: 1766-9$

20. List AF, Garewal HS, Sandberg AA. The myelodysplastic syndromes: biology and implications for management. J Clin Oncol 1990; 8 : 1424-41.

21. Cheson BD. The myelodysplastic syndromes : current approaches to therapy. Ann Intern Med 1990 ; 112 : 932-41.

22. Vadhan-Raj S, Keating M, LeMaistre A, et al. Effects of recombinant human granulocyte-macrophage colony-stimulating factor in patients with myelodysplastic syndromes. $N$ Engl J Med 1987; 317 : 1545-52.

23. Antin JH, Smith BR, Holmes W, et al. Phase I/II study of recombinant human granulocyte-macrophage colony-stimulating factor in aplastic anemia and myelodysplastic syndrome. Blood 1988; 72 : 705-13.

24. Ganser A, Völkers B, Greher J, et al. Recombinant human granulocytemacrophage colony-stimulating factor in patients with myelodysplastic syndromes. A phase I/II trial. Blood 1989; 73 : 31-7.

25. Vadhan-Raj S, Buescher S, LeMaistre A, et al. Stimulation of hematopoiesis in patients with bone marrow failure and in patients with malignancy by recombinant human granulocyte-macrophage colonystimulating factor. Blood $1988 ; 72$ : 134-41.

26. Hittelman WN, Tigaud JD, Estey E, et al. Relative maturation of aneuploid and diploid elements in patients with myelodysplasia treated with granulocyte-macrophage colony-stimulating factor. Blood 1988; 72 (suppl 1) : 121a.

27. Vadhan-Raj S, Broxmeyer HE, Spitzer G, et al. Stimulation of nonclonal hematopoiesis and suppression of the neoplastic clone after treatment with recombinant human granulocyte-macrophage colonystimulating factor in a patient with therapyrelated myelodysplastic syndrome. Blood 1989 ; $74: 1491-8$.
- Effet de potentialisation. La mesure par cytométrie en flux du contenu cellulaire en $\mathrm{ADN}$ et en ARN montre que le G-CSF, le GM-CSF et l'IL-3 augmentent le pourcentage de cellules en phase $\mathrm{S}$ et le passage de cellules de la phase $G_{0}$ vers la phase $G_{1} d u$ cycle cellulaire. Ces données font supposer que ces facteurs de croissance peuvent sensibiliser les cellules blastiques aux agents antimitotiques dépendants du cycle. De fait, la différenciation de la lignée $\mathrm{KG} 1$ induite par de faibles concentrations d'aracytine $\left(10^{-7} \mathrm{~mol} / \mathrm{l}\right)$ est nettement augmentée par traitement préalable par du G-CSF ou du GMCSF. Cet effet amplificateur est retrouvé à concentration cytotoxique $\left(10^{-3} \mathrm{~mol} / \mathrm{l}\right)$ d'aracytine avec une forte corrélation entre l'augmentation du nombre de morts cellulaires et l'augmentation du pourcentage de cellules en phase $\mathrm{S}$ ou entrant dans le cycle [7]. Des résultats similaires ont été rapportés avec l'IL-3 [17].

- Effet de stimulation de l'hématopoïse normale. Les facteurs de croissance sont déjà utilisés dans les LAM après la chimiothérapie, non plus pour leur effet de potentialisation de cette dernière, mais pour accélérer la reprise de l'hématopoïèse et réduire le temps d'aplasie pendant lequel surviennent la plupart des décès infectieux. Ainsi, les durées d'aplasie - après chimiothérapies associant soit daunorubicine, aracytine et 6 -thioguanine pour les LAM de novo, chez des patients âgés de plus de 65 ans, soit aracytine et mitoxantrone pour les leucémies aiguës en rechute - ont été analysées après ou en l'absence d'administration de GM-CSF. La comparaison entre le groupe des patients ayant reçu du GM-CSF à partir du 3e jour après chimiothérapie et le groupe historique contrôle ayant reçu la même chimiothérapie montre que le temps d'aplasie est diminué de 10 jours pour les patients âgés, et de 6 jours pour les patients en rechute. On ne dispose pas encore des résultats en termes de nombre et de gravité des infections et de taux de rémission complète [18]. Inversement, l'équipe du M.D. Anderson Cancer Center a administré du GM-CSF à 12 patients atteints de LAM, non préalablement traités mais à haut risque de décès pendant l'aplasie, sans bénéfice par rapport à une série historique, ni en termes de mortalité, ni en termes de durée d'aplasie [19].

Ainsi, les facteurs de croissance hématopoïétiques sont susceptibles d'entrer dans l'arsenal thérapeutique des leucémies aiguës pour leur effet de potentialisation des chimiothérapies cytotoxiques ou pour leur effet d'activation de la reconstitution hématopoiétique. Toutefois, c'est avec la plus grande circonspection que les études cliniques sont menées, tant le risque d'accélérer la prolifération blastique sans pouvoir la contrôler impose une sélection stricte des patients à inclure.

Syndromes myélodysplasiques Ces hémopathies, souvent primitives mais parfois secondaires à l'exposition à des agents alkylants (benzène, chimiothérapie), sont caractérisées par une anomalie qualitative et quantitative des cellules myéloïdes et par un risque élevé d'évolution vers une leucémie vraie. En l'absence de traitement efficace [20, 21], les données de laboratoire montrant que le GCSF pouvait induire la différenciation de cellules myéloïdes normales et leucémiques [14] ont conduit à l'utilisation des CSF dans ce cadre pathologique. L'administration en perfusion intraveineuse continue pendant 14 jours de GM-CSF à des patients neutropéniques a conduit, dans l'étude du M.D. Anderson Cancer Center, à une augmentation du nombre des globules blancs (de 5 à 70 fois plus nombreux après traitement qu'avant), des polynucléaires (de 5 à 373 fois), des éosinophiles, des monocytes et aussi des lymphocytes. Le traitement d'entretien n'a permis d'obtenir un effet sur les lignées rouge et plaquettaire que pour trois patients sur huit avec arrêt des besoins transfusionnels pour deux patients sur trois [22]. D'autres études confirment ces résultats [23, 24], bien que la remontée plaquettaire ou la correction de l'anémie paraissent peu fréquentes et modérées [24, 25]. La stimulation de la prolifération blastique médullaire, risque potentiel du GM-CSF, a été particulièrement bien analysée : ainsi, l'étude du M.D. Anderson Cancer Center n'a pas objectivé de prolifération ou de différenciation préférentielles du clone tumoral, démonstration apportée en 
Tableau I

CARACTÉRISTIQUES DES FACTEURS DE CROISSANCE HÉMATOPOÏÉTIQUES

\begin{tabular}{|c|c|c|c|c|c|}
\hline de & $\begin{array}{l}\text { Facteur } \\
\text { croissance }\end{array}$ & $\begin{array}{c}\text { Masse } \\
\text { moléculaire (kDa) }\end{array}$ & $\begin{array}{l}\text { Localisation } \\
\text { chromosomique }\end{array}$ & $\begin{array}{l}\text { Source } \\
\text { cellulaire }\end{array}$ & Cellules cibles \\
\hline & IL-3 & $14-28$ & $5 q 23-31$ & Lymphocyte T & $\begin{array}{c}\text { CFU-blast, CFU-GEMM, CFU-GM } \\
\text { CFU-G, CFU-M, CFU-Eo, CFU-Meg } \\
\text { CFU-Baso, BFU-E, PN éosinophiles, } \\
\text { monocytes }\end{array}$ \\
\hline & GM-CSF & 14-35 & $5 q 23-31$ & $\begin{array}{c}\text { Lymphocyte T } \\
\text { Monocyte } \\
\text { Fibroblaste } \\
\text { Cellule endothéliale }\end{array}$ & $\begin{array}{c}\text { CFU-blast, CFU-GEMM, CFU-GM } \\
\text { CFU-G, CFU-M, CFU-Eo, CFU-Meg } \\
\text { BFU-E, PN neutrophiles } \\
\text { PN éosinophiles, monocytes }\end{array}$ \\
\hline & G-CSF & $18-22$ & $17 q 11.2-21$ & $\begin{array}{c}\text { Monocyte } \\
\text { Fibroblaste } \\
\text { Cellule endothéliale }\end{array}$ & CFU-G, PN neutrophiles \\
\hline & M-CSF & $70-90$ & $5 q 33.1$ & $\begin{array}{c}\text { Monocyte } \\
\text { Fibroblaste } \\
\text { Cellule endothéliale }\end{array}$ & CFU-M, monocytes \\
\hline & EPO & 34-39 & $7 q 11-22$ & $\begin{array}{c}\text { Cellule péritubulaire } \\
\text { du rein } \\
\text { Cellule de Küpfer }\end{array}$ & BFU-E tardif, CFU-E, CFU-Meg \\
\hline
\end{tabular}

Pour la signification des abréviations, voir glossaire p. 445 .

recherchant, dans les polynucléaires du sang périphérique, les anomalies cytogénétiques caractérisant l'hémopathie par induction de la condensation prématurée des chromosomes [26]. En revanche, une étude allemande a retrouvé une augmentation des blastes sanguins ou médullaires chez sept patients parmi les 11 traités par GM-CSF dans le cadre d'un essai de phase I/II. Quinze pour cent serait le seuil d'infiltration blastique médullaire au-dessus duquel la prescription de GM-CSF risquerait de faire progresser la maladie vers la leucémie vraie [24], mais d'autres études sont encore nécessaires pour préciser ce point. Au contraire, le traitement par le GM-CSF d'une patiente atteinte d'anémie réfractaire avec excès de blastes, secondaire à une chimiothérapie pour cancer de l'ovaire, a permis d'obtenir la rémission hématologique complète pendant près d'un an après l'arrêt du traitement. Cette rémission a été documentée par des études cytologiques et cytogénétiques (chromosomes métaphasiques sur moelle et chromosomes prématurément condensés du sang $m / s n^{\circ} 5$, vol. 7 , mai 91 périphérique), ainsi que des caractéristiques de croissance in vitro des progéniteurs myéloïdes (erythroid burst forming units (BFU-E) et granulocytemacrophage colony forming units (CFU$\mathrm{GM})$ ) et du polymorphisme des fragments de restriction des chromosomes X [27]. A noter, enfin, que la stimulation par le GM-CSF des fonctions de ses cellules effectrices normales [28] justifie les études en cours sur la nature, l'intensité et le retentissement clinique de cette action sur les cellules myélodysplasiques. Le G$\mathrm{CSF}$, pour lequel on dispose de nettement moins d'études cliniques, semble augmenter le nombre des leucocytes et des polynucléaires selon un mode dépendant de la dose [29]; une diminution sous traitement du besoin transfusionnel a même été notée dans quelques observations [30].

L'IL-3 a plus récemment été étudiée chez les patients porteurs de myélodysplasie : dans le cadre d'un essai allemand de phase I-II, neuf patients atteints de ce syndrome ont reçu de 250 à $500 \mu \mathrm{g} / \mathrm{m}^{2}$ d'IL-3 en traitement sous-cutané de 15 jours ; le nombre de leucocytes a augmenté chez tous les patients dans un rapport de 1,3 à 3,6 par rapport aux chiffres préthérapeutiques. Deux patients sur quatre, très thrombopéniques, ont eu une réponse plaquettaire suffisante pour que puissent être interrompues les transfusions plaquettaires. Les besoins érythrocytaires ont diminué chez un patient. Enfin, il a été noté une augmentation significative des taux sériques d'immunoglobulines $\mathrm{M}$ et $\mathrm{A}$, témoignant d'une stimulation des plasmocytes. En revanche, la maladie a progressé chez un patient dont l'infiltration blastique médullaire et sanguine a augmenté [31].

Une étude pilote d'EPO à forte dose a été rapportée par une équipe suisse : un patient sur les deux atteints de myélodysplasie et un sur les deux atteints d'hémoglobinurie paroxystique nocturne ont vu leur besoin transfusionnel diminué de façon significative après administration d'EPO en intraveineux trois fois par semaine [32].

Ainsi, l'intérêt des facteurs de croissance dans les syndromes myélodys- 


\section{RÉFÉRENCES}

28. Ruef C, Coleman DL. Granulocytemacrophage colony-stimulating factor : pleiotropic cytokine with potential clinical usefulness. Rev Infect Dis 1990; 12 : 41-62.

29. Kobayashi Y, Okabe T, Ozawa K, et al. Treatment of myelodysplastic syndromes with recombinant human granulocyte colony-stimulating factor: a preliminary report. Am J Med 1989 ; 86 : 178-82.

30. Negrin RS, Haeuber DH, Nagler A, et al. Treatment of myelodysplastic syndromes with recombinant human granulocyte colony-stimulating factor : a phase I/II trial. Ann Intern Med 1989; 110 : 976-84.

31. Ganser A, Seipelt G, Lindemann A, et al. Effects of recombinant human interleukin 3 in patients with myelodysplastic syndromes. Blood $1990 ; 76$ : 455-62.

32. Stebler C, Tichelli A, Dazzi H, et al. High-dose recombinant human erythropoietin for treatment of anemia in myelodysplastic syndromes and paroxysmal nocturnal hemoglobinuria : a pilot study. Exp Hematol $1990 ; 18$ : 1204-8.

33. Demetri GD, Griffin JD. Hematopoietic growth factors and high-dose chemotherapy: will grams succeed where milligrams fail. J Clin Oncol 1990; 8 : 761-4.

34. Bronchud MH, Scarffe JH, Thatcher N, et al. Phase I/II study of recombinant human granulocyte colony-stimulating factor in patients receiving intensive chemotherapy for small cell lung cancer. $\mathrm{Br} J \mathrm{Can}$ cer $1987 ; 56$ : 809-13.

35. Gabrilove JL, Jakubowski A, Scher H, et al. Effect of granulocyte colony-stimulating factor on neutropenia and associated morbidity due to chemotherapy for transitionalcell carcinoma of the urothelium. $N$ Engl J Med 1988 ; 312 : 1414-22.

36. Gabrilove JL, Jakubowski A, Fain K, et al. Phase I study of granulocyte colonystimulating factor in patients with transitional-cell carcinoma of the urothelium. J Clin Invest 1988 ; 82 : 1454-61.

37. Morstyn G, Campbell L, Souza LM, et al. Effect of granulocyte colony-stimulating factor on neutropenia induced by cytotoxic chemotherapy. Lancel 1988 ; 1 : 667-72.

38. Antman KS, Griffin JD, Elias A, et al. Effect of recombinant human granulocytemacrophage colony-stimulating factor on chemotherapy-induced myelosuppression. $N$ Engl J Med 1988; 319 : 593-8.

39. Barlogie B, Jagannath S, Dixon DO, et al. High-dose melphalan and granulocytemacrophage colony-stimulating factor for refractory multiple myeloma. Blood 1990 ; $76: 677-80$.

40. Berdel WE, Danhauser-Riedl S, Steinhauser $\mathrm{G}$, el al. Various human hematopoietic growth factors (interleukin 3, GM-CSF G-CSF stimulate clonal growth of nonhematopoietic tumor cells. Blood 1989 ; plasiques paraît d'ores et déjà clair. Les objectifs actuels sont une meilleure définition du risque de prolifération blastique sous traitement et la mise en œuvre de protocoles associant différents facteurs de croissance de sorte que diminuent les risques infectieux, hémorragiques, ceux liés à l'anémie, et que s'améliore la survie.

\section{Cytopénies induites par les chimiothérapies anticancéreuses}

Réduire la durée et la profondeur des neutropénies secondaires aux chimiothérapies s'est vite imposé comme l'une des indications potentielles majeures des CSF [33]. Le risque infectieux lié à la neutropénie fait de la toxicité hématologique le facteur limitant de l'augmentation des doses d'antinéoplasiques. Le bénéfice des CSF peut s'exprimer en termes de réduction de la morbidité et de la mortalité liées aux infections, mais aussi en termes de respect des doses optimales d'antinéoplasiques et des intervalles entre les traitements.

\section{Chimiothérapies à doses conven- tionnelles}

Une équipe anglaise a initialement rapporté l'effet bénéfique du G-CSF chez 12 patients recevant une polychimiothérapie associant doxorubicine, ifosfamide et étoposide pour des cancers du poumon à petites cellules : non seulement la durée et le nadir* de la neutropénie sont moins prononcés en présence de G-CSF, mais encore, observe-t-on moins d'épisodes fébriles [34]. De même, un essai de phase I/II a été conduit en administrant de 1 à $60 \mu \mathrm{g} / \mathrm{kg} / \mathrm{jour}$ de G-CSF après la première cure de chimiothérapie de type M-VAC associant méthotrexate, vinblastine, doxorubicine et cisplatine à 27 patients atteints d'un cancer de la vessie. Une augmentation, dépendante de la dose de G-CSF, du nombre des polynucléaires a été observée, aboutissant à une diminution significative du nombre de jours d'administration d'antibiotiques ainsi que de l'incidence et de la gravité des mucites buccales. Une conséquence

* Nadir : point le plus bas, opposé au zénith. importante a été la possibilité d'administration de la cure suivante de chimiothérapie au jour programmé à tous les patients recevant le G-CSF, tandis qu'elle fut repoussée chez plus de $70 \%$ des patients ne le recevant pas. Ces données ne se traduisent pour l'instant ni par une amélioration des taux de réponse aux chimiothérapies ni, a fortiori, par une augmentation des taux de guérison, mais ils sont encourageants en termes de diminution de la toxicité et d'amélioration de la qualité de vie $[35,36]$. De nombreuses autres tumeurs à des stades avancés ont été traitées par une association de melphalan et de G-CSF avec un résultat similaire aux précédents en termes de réduction de la neutropénie [37]. Le GM-CSF a été utilisé dans le même but chez 16 patients atteints de sarcomes et traités par une polychimiothérapie associant doxorubicine, if osfamide et dacarbazine. Les cures ont pu être rapprochées en raison du raccourcissement de la période de neutropénie [38]. Une étude très intéressante de traitement de myélome multiple réfractaire par $100 \mathrm{mg} / \mathrm{m}^{2}$ de melphalan suivis de 0,25 à $0,75 \mathrm{mg} / \mathrm{m}^{2}$ de GM-CSF par jour en sous-cutané a été rapportée. La comparaison avec une série contrôle historique de 43 patients montre que le bénéfice du GM-CSF s'exprime essentiellement chez les patients jeunes n'ayant été que peu traités préalablement, et ayant donc probablement conservé une certaine réserve médullaire [39].

Si le risque de prolifération blastique sous traitement des LAM par des CSF hématopoïétiques est très clair, la question est aussi posée pour les tumeurs solides. En effet, l'IL-3, le G-CSF et le GM-CSF peuvent stimuler la croissance des cellules clonogéniques de différentes lignées cellulaires d'adénocarcinomes coliques humains (HTB-38, CCL 187) [40]. Par ailleurs, l'effet de l'IL-3, du GM-CSF et du G-CSF sur la formation in vitro de colonies obtenues à partir de tumeurs humaines solides d'histologie variée a été testé. L'analyse des seules tumeurs pour lesquelles un développement satisfaisant de la croissance tumorale avait été obtenu en l'absence de CSF hématopoïétiques montre que

$$
m / s n^{\circ} 5 \text {, vol. } 7 \text {, mai } 91
$$


l'adjonction de l'IL-3 stimule de façon significative 10 tumeurs sur 44 , celle de GM-CSF cn stimule 8 sur 43 et celle de G-CSF, 6 sur 40 [41]. Ces données in vitro n'ont pour l'heure pas encore fait l'objet de confirmation in vivo, mais doivent inciter à une appréciation très détaillée de l'évolution du volume tumoral des patients recevant des CSF hématopoïétiques après chimiothérapie. Il en est de même pour le myélome multiple: l'effet du GM-CSF sur la croissance tumorale a été analysé in vitro chez 21 patients dont 17 avaient des tumeurs considérées comme proliférantes, avec plus de $1 \%$ des cellules myélomateuses en phase $\mathrm{S}$. Le GM-CSF a augmenté la prolifération induite par l'IL-6 de ces dernières, et a entraîné une production locale de GM-CSF par le microenvironnement médullaire [42].

L'anémie est une complication fréquente des affections cancéreuses et de leurs traitements. Aussi l'EPO at-elle été testée par une équipe autrichienne chez 13 patients suivis pour myélome multiple, le plus souvent traités depuis longtemps, tous par chimiothérapie et onze par une association de radiothérapie et de chimiothérapie. Seul un patient avait une insuffisance rénale, mais tous étaient anémiques avec une hémoglobine inférieure à $110 \mathrm{~g} / \mathrm{l}$ et des symptômes fonctionnels liés à cette anémie. Chaque patient a reçu 150 unités/kg en sous-cutané trois fois par semaine avec possibilité d'augmentation progressive de la dose en cas d'échec. La chimiothérapie ou la radiothérapie (ou les deux) ont été poursuivies chez neuf patients pendant l'administration de l'EPO. Onze malades ont répondu au traitement selon le critère prédéfini d'une augmentation d'au moins $20 \mathrm{~g} / \mathrm{l}$ du taux d'hémoglobine sans transfusion. Les réponses survinrent avec un délai moyen de cinq semaines. Par ailleurs, tous les patients répondeurs ont vu soit disparaître, soit diminuer nettement les signes cliniques de l'anémie avec comme résultante une amélioration significative du performance status après deux mois de traitement [43]. Ainsi, l'EPO est susceptible de rentrer dans l'arsenal thérapeutique en oncologie : en réduisant les besoins transfusionnels, on peut en attendre une amé$\mathrm{m} / \mathrm{s} n^{\circ}$ 5, vol. 7, mai 91 lioration du confort de vie des patients, une meilleure tolérance aux traitements et, bien sûr, une diminution des complications liées aux transfusions.

Intensifications thérapeutiques suivies de greffe de moelle

Les chimiothérapies massives suivies de reconstitution médullaire par autogreffe de moelle permettent d'utiliser l'effet-dose des chimiothérapies, au prix d'une toxicité hématologique importante que l'on tente de réduire avec les CSF. Ainsi, après traitement par autogreffe de lymphomes malins non hodgkiniens, l'administration de $60 \mu \mathrm{g} / \mathrm{m}^{2} /$ jour de GM-CSF a permis de réduire la durée des neutropénies inférieures à $0,5 \times 10^{\circ} \mathrm{PN} / \mathrm{l}$ de 14 à 25 jours, de ramener la dernière transfusion plaquettaire du $38^{\mathrm{e}}$ au $29^{e}$ jour après greffe, et de diminuer le nombre de jours de fièvre de 12 à 6 et le nombre de jours d'hospitalisation de 41 à 29 [44]. Dans le cas de tumeurs solides réfractaires, le GM-CSF utilisé après autogreffes associées à un conditionnement médullaire associant cyclophosphamide, carmustine et cisplatine, a diminué l'incidence des épisodes septicémiques alors même que la durée d'aplasie n'a pas été significativement diminuée [45]. Le G-CSF a été utilisé dans les mêmes conditions avec des résultats similaires chez des patients atteints de maladie de Hodgkin et traités par autogreffe [46].

On ne dispose pas encore, à l'heure actuelle, d'information précise sur l'effet des CSF après transplantation allogénique de moelle, car le risque potentiel de stimulation des cellules médiatrices de la réaction du greffon contre l'hôte $(\mathrm{GVH})$ ou du rejet du greffon freine la mise en œuvre des essais thérapeutiques dans cette indication [47]. Le GM-CSF a toutefois été administré à fortes doses (de 60 à $1000 \mu \mathrm{g} / \mathrm{m}^{2} /$ jour), en l'absence de prise, à 15 patients ayant reçu une greffe allogénique rejetée : il n'a pas été vu d'exacerbation de la réaction de GVH chez les neuf patients considérés comme répondeurs au GM-CSF (patients ayant plus de $0,5 \times 10^{9}$ $\mathrm{PN} / \mathrm{l}$ dans les 14 jours suivant le début du traitement par GM-CSF) [48].

Le bénéfice apporté par l'effet-dose que permettent les chimiothérapies massives associées ou non à une radiothérapie est de toute façon conditionné par l'obtention de cellules souches totipotentes non contaminées par des cellules tumorales: la fréquence des atteintes médullaires au cours des tumeurs malignes, hématologiques ou non, a conduit à développer les reconstitutions hématologiques à partir de cellules souches totipotentes collectées dans le sang périphérique par cytaphérèse [49]. La constatation d'une augmentation du nombre de ces cellules souches totipotentes hématopoïétiques dans le sang périphérique sous traitement par les CSF [50] a permis d'utiliser ces derniers pour restaurer l'hématopoï̀se après traitement antitumoral à dose massive. Ainsi, 12 patients atteints d'hémopathies malignes non myéloïdes (11 cas) ou de sarcome (1 cas) - dont les moelles étaient devenues très hypocellulaires en raison de traitements antérieurs - ont $\mathrm{vu}$, après administration de GM-CSF pendant une moyenne de 11,5 jours, leurs CFU-GM (granulocyte-macrophage colony forming units) passer en moyenne de $158,5 / \mathrm{ml}$ à $1347 / \mathrm{ml}$ après. Parallèlement, chez sept patients, la concentration des BFU$\mathrm{E}$ (erythroid burst forming units) a été multipliée en moyenne par 15,2. L'intensification thérapeutique a pu être réalisée chez six patients dont cinq ont eu une reconstitution hématologique des trois lignées : leucocytaire (plus de $1,0 \mathrm{~GB} / \mathrm{nl}$ en 15 jours et plus de $0,5 \mathrm{PN} / \mathrm{nl}$ en 28,5 jours en moyenne), érythrocytaire et plaquettaire (plus de 20 plaquettes/nl en 39 jours en moyenne). Le sixième patient est en fait rapidement décédé d'une reprise évolutive du lymphome malin non hodgkinien pour lequel il était suivi, avec un envahissement médullaire qui le rend ininterprétable pour cette étude [51]. Toutefois, il est bien clair que la combinaison des CSF et de la collecte des cellules souches totipotentes circulantes par cytaphérèse est une voie essentielle dans l'avenir pour les intensifications thérapeutiques.

\section{Effets secondaires} des facteurs de croissance

En pratique, les effets secondaires des CSF sont assez modestes. Des dou- 


\section{RÉFÉRENCES}

41. Joraschkewitz $M$, Depenbrock $H$ Freund $M$, et al. Effects of cytokines on in vitro colony formation of primary human tumor specimens. Eur.J Cancer 1990; 26 : $1070-4$.

42. Zhang XG, Bataille $R$, Jourdan $M$, et al. Granulocyte-macrophage colonystimulating factor synergizes with interleukin 6 in supporting the proliferation of human myeloma cells. Blood 1990; 76 : 2599-6()5

43. Ludwig H, Fritz li, Kotzmann $\mathrm{H}$, el al. Erythropoietin treatment of anemia associated with multiple myeloma. $N$ Engl J Med 1990 : 322 : 1693-9.

44. Nemunaitis J, Singer JW, Buckner CI), 't al. Use of recombinant human granulocyte-macrophage colony-stimulating factor in autologus marrow transplantation for lymphoid malignancies. Blood $1988 ; 72$ : 834-6.

45. Brandt SJ, Peters WP, Atwater SK, et al. Effect of recombinant human granulocyte-macrophage colony-stimulating factor on hematopoietic reconstitution after high-dose chemotherapy and autologus bone marrow transplantation. $\Lambda^{\prime}$ Engl $J$ Med $1988 ; 318: 869-76$.

46. Taylor KM, Jagannath S, Spitzer G, it al. Recombinant human granulocyte colons-stimulating factor hastens granulocyte recovery after high-dose chemotherapy and autologus bone marrow transplantation in Hodgkin's discase. J Cilin Oncol 1989; 7 1791-9.

t7. Mertelsmann R, Herrmann F, Hecht 'T, at al. Hematopoietic growth factors in bone matrow transplantation. Bone Marrow Transplant 1990; 6: 73-7.

48. Nemunaitis J, Singer JW, Buckner CD, it al. Use of recombinant human granu locyte colony-stimulating factor in yraft failure after bone marrow transplantation. Blood 1990 ; 76 : 245-53

49. Kessinger A, Armitage JO, Landmark JD, et al. Reconstitution of human hematopoietic function with autologus cryopreserved circulating stem cells. Exp Hematol $1986 ; 14: 192-6$.

50. Socinski MA, Cannistra SA, Elias A, 't al. Granulocyte-macrophage colonystimulating factor expands the circulating haemopoietic progenitor cell compartment in man. Lancet 1988; 1 : 1194-8.

51. Haas R, Dick Ho A, Bredthauer U, $e^{\prime}$ al. Successful autologus transplantation of blood stem cells mobilized with recombinan human granulocyte-macrophage colonystimulating factor. Exp Hematol $1990 ; 18$ 94-8.

52. Groopman Jl:, Molina JM, Scadden DT. Hematopoietic growth factors: biology and clinical applications. $N$ Engl.J Med 1989; 321 : 1449-59

53. Morstyn G, Lieschke GJ, Sheridan W, et al. Clinical experience with recombinan human granulocyte colony-stimulating factor and granulocyte-macrophage colonystimulating factor. Semin Hematol $1989 ; 26$ lcurs osscuses ont été observées au cours de traitement par G-CSF ou par GM-CSF ; elles dépendent de la dose administrée et sont réversibles à l'arrêt du traitement. De la fièvre avec asthénic et anorexie, des myalgies et des arthralgics ont été plus fréquemment décrites avec le GM-CSF qu'avec le G$\mathrm{CSF}$, de même qu'une éruption nummulaire érythémateuse ou purpurique au point d'injection sous-cutané. Ce n'est qu'à fortes doses de GM-CSF qu'ont été vus des syndromes de fuite capillaire avec épanchements pleuraux, péricardiques et ascitiques ou des thromboses des gros vaisseaux. Ces effets secondaires graves peuvent être rattachés soit au GM-CSF lui-même, soit à un effet indirect passant par la libération de TNF ou d'IL-1. Un effet secondaire immédiat a été rapporté dans de nombreux cas lors de la première administration de GM-CSF : 30 minutes après l'administration du produit, on pcut observer une baisse du nombre des polynucléaires dans le sang périphérique, associéc à une baisse de la saturation en oxygène et à une discrète baisse tensionnclle. La libération de substances vasodilatatrices est supposée en être le mécanisme. Enfin, un syndrome de Swect, avec dermite aiguë fébrile à polynucléaires ncutrophiles, a été décrit au cours d'un traitement par G-CSF [52, 53].

\section{Conclusion}

Les facteurs de croissance hématopoïétiques se sont très vite imposés comme un élément essentiel de l'arsenal thérapeutique en oncohématologie : en stimulant l'hématopoïèse, ils peuvent améliorer les insuffisances médullaires des syndromes myélodysplasiques, potentialiser les chimiothérapies des leucémies aiguës et en diminuer la toxicité, faciliter l'administration des chimiothérapies antinéoplasiques et la sortic d'aplasie après greffe de moelle ou encore améliorer le confort de vie de patients. De nombreux travaux sont en cours pour en déteminer les meillcurs schémas d'administration, reposant notamment sur l'association de différents CSF

\section{TIRÉS A PART}

\section{J.-D. Tigaud.}

\section{Summary}

Clinical applications of hematopoietic colony-stimulating factors in onco-hematology

The major hematopoictic growth factors have been produced through recombinant DNA technology and the preliminary results of many clinical trials are already published. The hematopoietic colony-stimulating factors might be useful in therapy of acute mycloid leukemias cither by potentialization of antileukemic therapies or by fastening recovery from aplasia. Granulocytemacrophage colony-stimulating factor (GM-CSF), granulocyte colony-stimulating factor (G-CSF), interleukin 3 and erythropoietin have been given to patients with myclodysplastic syndromes and improved their white blood cell counts but had a very mild and transitory effect on platelets and red cells. Patients with solid tumors (i.c. small cell carcinoma of the lung or bladder cancer) have been given, usually with success, G-CSF or GM-CSF in order to reduce the toxicity of chemotherapy and to improve the drugs administration schedulc. Erythropoictin may be an effective agent to prevent or correct anemia of cancer patients. After bone marrow transplantation, the neutropenia duration has been decreased by G-CSF or GM-CSF without secondary effect such as exacerbation of the graft-versus-host disease. Indeed, combination of different growth factors acting at different steps of cellular differentiation (i.e. IL-3 and GM-CSF) has already entered clinical trials. 